\title{
Lusioersily
}

\section{On children's variable success with scalar inferences: Insights from disjunction in the scope of a universal quantifier}

Pagliarini, E., Bill, C., Romoli, J., Tieu, L., \& Crain, S. (2018). On children's variable success with scalar inferences: Insights from disjunction in the scope of a universal quantifier. Cognition, 178(0), 178-192. https://doi.org/10.1016/j.cognition.2018.04.020

Link to publication record in Ulster University Research Portal

Published in:

Cognition

Publication Status:

Published (in print/issue): 30/09/2018

DOI:

10.1016/j.cognition.2018.04.020

\section{Document Version}

Author Accepted version

\section{General rights}

Copyright for the publications made accessible via Ulster University's Research Portal is retained by the author(s) and / or other copyright owners and it is a condition of accessing these publications that users recognise and abide by the legal requirements associated with these rights.

\section{Take down policy}

The Research Portal is Ulster University's institutional repository that provides access to Ulster's research outputs. Every effort has been made to ensure that content in the Research Portal does not infringe any person's rights, or applicable UK laws. If you discover content in the Research Portal that you believe breaches copyright or violates any law, please contact pure-support@ulster.ac.uk. 


\title{
On children's variable success with scalar inferences: Insights from disjunction in the scope of a universal quantifier
}

\author{
Elena Pagliarini ${ }^{*}$, Cory Bill ${ }^{2}$, Jacopo Romoli ${ }^{3}$, Lyn Tieu ${ }^{4}$, and \\ Stephen Crain ${ }^{2}$
}

${ }^{1}$ Center for Brain and Cognition, Departament de Tecnologies de la Informació i les Comunicacions, Universitat Pompeu Fabra, calle Ramon Trias Fargas 25-27, 08005 Barcelona, Spain

${ }^{2}$ ARC Centre of Excellence in Cognition and its Disorders, Australian Hearing Hub, 16 University Avenue, Macquarie University, 2109, NSW, Australia

${ }^{3}$ School of Communication, Ulster University, Jordanstown campus, Shore Road, Newtownabbey Co. Antrim BT37 0QB UK ${ }^{4}$ Western Sydney University, Locked Bag 1797, Penrith NSW 2751 Australia

\begin{abstract}
Previous developmental studies have revealed variation in children's ability to compute scalar inferences. While children have been shown to struggle with standard scalar inferences (e.g., with scalar quantifiers like "some") (Noveck 2001, Chierchia et al. 2001, Papafragou and Musolino 2003, Guasti et al. 2005), there is also a growing handful of inferences that children have been reported to derive quite readily (Papafragou and Musolino 2003, Barner and Bachrach 2010, Stiller et al. 2015, Tieu et al. 2016. Singh et al. 2016 | Hochstein et al. 2016, Tieu et al.|2017). One recent approach, which we refer to as the Alternatives-based approach, attributes the variability in children's performance to limitations in how children engage with the alternative sentences that are required to compute the relevant inferences. Specifically, if the alternative sentences can be generated by simplifying the assertion, rather than by lexically replacing one scalar term with another, children should be better able to compute the inference. In this paper, we investigated this prediction by assessing how children and adults interpret sentences that embed disjunction under a universal quantifier, such as "Every elephant caught a big butterfly or a small butterfly". For adults, such sentences typically give rise to the distributive inference that some elephant caught a big butterfly and some
\end{abstract}


elephant caught a small butterfly (Gazdar 1979, Fox 2007, Crnič et al. 2015). Another possible interpretation, though not one typically accessed by adults, is the conjunctive inference that every elephant caught a big butterfly and a small butterfly (Singh et al. 2016). Crucially, for our purposes, it has been argued that both of these inferences can be derived using alternatives that are generated by deleting parts of the asserted sentence, rather than through lexical replacement, making these sentences an ideal test case for evaluating the predictions of the Alternatives-based approach. The findings of our experimental study revealed that children are indeed able to successfully compute this class of inferences, providing support for the Alternatives-based approach as a viable explanation of children's variable success in computing scalar inferences.

Keywords: implicatures, language acquisition, distributive inference, conjunctive inference, pragmatics, disjunction, universal quantification

\section{Introduction}

According to the standard semantics of disjunction, which is derived from classical propositional logic, a basic disjunctive sentence like (1) is true if at least one of its disjuncts is true. However, when a sentence like (1) is used in everyday conversation, it often gives rise to the additional inferences in (2) and (3), sometimes called scalar inferences ${ }^{1}$ (Gazdar 1979, Sauerland 2004, Fox 2007. among many others).

(1) The elephant caught a big butterfly or a small butterfly.

(2) The elephant didn't catch both a big butterfly and a small butterfly.

(3) The speaker is ignorant as to whether the elephant caught a big butterfly and as to whether the elephant caught a small butterfly.

The traditional Gricean explanation for how such inferences are derived involves a combination of general reasoning and assumptions about how rational agents interact in conversation (Grice 1975, Gamut 1991, among many others). More specifically, the proposal is that the hearer assumes that at any given point during a conversation, the speaker will produce the most informative utterance that is relevant for the purposes of the conversation and that she believes to be true. For example, when the speaker utters (1), the hearer will reason that if the speaker could have uttered the more informative sentence (4), then she would have done so. The fact that the speaker uttered (1), rather than (4), leads the hearer to infer that the speaker does not have sufficient evidence that (4) is true. If the hearer takes the speaker to be well-informed, then the hearer is led to infer the negation of (4), which is the exclusivity inference (2).

(4) The elephant caught a big butterfly and a small butterfly.

\footnotetext{
${ }^{1}$ Also known as scalar implicatures.
} 
The question of how such inferences are derived has been subject to much subsequent work since Grice and is the subject of ongoing debate. Approaches differ in whether the source of the phenomenon lies within the grammar or in a post-grammatical pragmatic domain. One aspect that most approaches agree on is that the hearer's reasoning about what the speaker might have said should be constrained to a set of alternatives (Horn 1972, Gazdar 1979, Katzir 2007, Fox and Katzir 2011). A standard idea in the literature is that at least some of these alternative sentences are generated on the basis of certain words being associated with others in the language user's mental lexicon. For example, Horn (1972) suggests that certain words occupy positions on lexical scales. The scales are ordered by informational strength (e.g., <or...and $>$, < some...all $>$, $<$ might...must $>$ ). ${ }^{2}$ When a speaker utters a sentence that includes one of the terms on the scale, alternative sentences are generated by replacing the relevant lexical item with one of its stronger scale-mates. For instance, the stronger alternative to the speaker's utterance (1) is sentence (4). The alternative sentence, (4), is generated by replacing the lexical item 'or' in (1) with the stronger scale-mate 'and.' Because the hearer supposes that the speaker is being cooperative and has produced the strongest statement that she was prepared to make, the hearer infers that sentence (1), with 'or', is the strongest statement the speaker felt she had evidence for. Therefore, the hearer infers the negation of the stronger alternative with 'and.' The negation of the alternative (4) yields the exclusivity inference 2 , 3

The relationship between the exclusivity inference and the ignorance inference (3) is controversial (for discussion, see Sauerland 2004, Fox 2007, Meyer 2013). One approach invokes the same mechanism that underlies the derivation of scalar inferences. On this approach, the ignorance inference (3) involves a comparison of the speaker's utterance (1) with the alternative sentences (5) and (6). These alternatives correspond to the individual disjuncts of the sentence in (1) Gazdar 1979, Sauerland 2004) 45 The alternative sentences (5) and (6) are both more informative than the asserted sentence (1). If the speaker had sufficient evidence that (5) and (6) were true, then she should have uttered these alternatives instead of (1). The fact that the speaker chose not to say (5) or (6) leads the hearer to infer that the speaker does not have sufficient evidence that

\footnotetext{
${ }^{2}$ The relative informativity of a lexical item on these scales is based on the entailment relationship sentences containing them. Sentence A is more informative than sentence B iff A asymmetrically entails B. For example, sentence 4 is more informative than (1) because (4) entails (1), but sentence (1) does not entail [4.

${ }^{3}$ More recent work on alternatives criticises this approach for merely stipulating which terms are associated with others in the lexicon, and argues instead for more general alternativegenerating algorithms for any given sentence. For discussion, see Katzir (2007), Fox and Katzir (2011), Trinh and Haida (2015), and Breheny et al. (2016).

${ }^{4}$ On certain accounts, the individual disjuncts from which ignorance inferences arise do not correspond to formal alternatives of a disjunctive sentence. Instead, they are simply more informative relevant sentence meanings the speaker might have said instead. For our purposes, it suffices that the ignorance inference can be derived without recourse to the lexical replacement of alternatives; we make no claims about the (non-)formal status of the alternatives in 5 and 6 .
} 
(5) or (6) are true, generating the ignorance inference in $(3) 5^{5}$

(5) The elephant caught a big butterfly.

(6) The elephant caught a small butterfly.

Note that there is no lexical replacement involved in generating the ignorance inference, because the alternatives in (5) and (6) correspond to the individual disjuncts of the asserted sentence (1) (Gazdar 1979, Sauerland 2004). That is, these alternatives can be generated through a simplification of the asserted sentence, specifically by deleting all but one of the disjuncts.

As we've now seen, the exclusivity and ignorance inferences can both be derived through reasoning over alternative sentences that the speaker might have uttered instead of what she actually said. Because of this, some accounts take theses inferences to be derived in the same way (e.g., Sauerland 2004). However, for reasons that we will discuss further, what is most relevant for our purposes is the distinction in the alternatives that are generated for each inference; one involves lexical replacement (i.e. the exclusivity inference), whereas the other involves simplification of the original assertion (i.e. the ignorance inference). Here and in the rest of the paper, we will refer to alternatives of the latter kind as non-replacement alternatives. For discussion regarding the distinction between alternatives generated by deletion versus alternatives generated by lexical replacement, see Katzir (2007) and Fox and Katzir (2011).

In addition to the substantial theoretical work on inferences like these, there has also been a great deal of experimental work, including developmental studies that assess children's ability to compute such inferences. The bulk of this work has reported that children consistently derive scalar inferences at lower rates than adults do (Noveck 2001, Chierchia et al. 2001, Papafragou and Musolino 2003. Guasti et al. 2005, Foppolo et al.|2012, among many others). However, this work has primarily focused on a rather restricted set of scalar inferences, typically those derived on the basis of alternatives that are generated through lexical replacement.

More recent investigations have expanded the range of inferences investigated to plurality inferences, free choice inferences, ad hoc implicatures, conjunctive inferences of disjunction, and ignorance inferences (Tieu et al.|2014, 2016, Stiller et al. 2015, Hochstein et al. 2016, Singh et al. 2016, Tieu et al. 2017). Some of these studies report low rates of inference derivation by children (Tieu et al. 2014), as in previous studies; however, others have reported adult-like rates of inference derivation by children (Tieu et al. 2016, Hochstein et al. 2016, Singh et al. 2016). Notably, the inferences that children have been reported to derive readily are ones that involve non-replacement alternatives.

One attempt to explain children's variable success in scalar inference computation is the Alternatives-based approach (Reinhart 2006, Barner et al. 2011, Tieu et al. 2016, Singh et al. 2016). On this approach, children's difficulties

\footnotetext{
${ }^{5}$ Unlike in the case above of the exclusivity inference, in this case the hearer cannot conclude that the speaker has evidence that (5) and (6) are false, as this would lead to a contradiction with the assertion; see Sauerland (2004) and Fox (2007) for discussion.
} 
in deriving the bulk of previously investigated scalar inferences, and their apparent success on a handful of other inferences, are explained by appealing to children's limitations in computing the relevant alternatives to the asserted sentence. Furthermore, the Alternatives-based approach makes clear predictions about children's behaviour in dealing with inferences that have not been investigated previously.

The present paper investigates the predictions of the Alternatives-based approach by investigating children's and adults' interpretations of sentences in which a disjunction is embedded under a universal quantifier, as in (7). Such sentences constitute an ideal case study for testing the Alternatives-based approach, because two of the inferences that arise from such sentences are based on non-replacement alternatives. Specifically, the sentence in $(7)$ licenses the distributive inference in (8) (Gazdar 1979, Crnič et al. 2015). It has also been proposed that children license the conjunctive inference in (9) (Fox 2007, Singh et al. 2016).

(7) Every elephant caught a big butterfly or a small butterfly.

(8) Some elephant caught a big butterfly and some elephant caught a small butterfly.

(9) Every elephant caught both a big butterfly and a small butterfly.

The derivation of the distributive inference in (8) involves the alternatives in (10) and (11), which are non-replacement alternatives as they can be generated by simplifying the asserted sentence in (7). As we will see, the findings of our experimental study reveal that children are adult-like in deriving such inferences. On the Alternatives-based approach, this finding is attributed to the fact that the relevant alternatives (i.e. (10) and (11)) are derived through simplification rather than lexical replacement.6

(10) Every elephant caught a big butterfly.

(11) Every elephant caught a small butterfly.

Moreover, we also found that a few children in our experiment computed the conjunctive inference in (9), as reported in previous developmental studies of children's interpretation of disjunction (Singh et al. 2016 for disjunction embedded under "every"; Paris 1973, Braine and Rumain 1981, Chierchia et al. 2004, Tieu et al. 2017 for unembedded disjunction).

To preview, we will conclude that the results of the present investigation are in line with the predictions of the Alternatives-based approach, providing further support for it as a viable explanation of children's variable success in computing a large class of scalar inferences.

The rest of the paper is organised as follows. First, we review previous developmental studies of scalar inferences. We then outline a prediction of

\footnotetext{
${ }^{6}$ The conjunctive inference is derived from slightly different alternatives; however, the alternatives are also generated through simplification of the disjunctive sentence. See Appendix C for details.
} 
the Alternatives-based approach, and present an experiment designed to test the prediction. We conclude with a discussion of how our results bear on the Alternatives-based approach.

\subsection{Children's variable performance on scalar inferences}

Children have been found to display variable success on scalar inferences. We will first consider some studies that have reported non-adult-like performance, and then move on to cases in which children were adult-like.

\subsubsection{Non-adult-like behaviour}

For over a decade, developmental research has found that children derive scalar inferences far less often than adults do (e.g., Noveck 2001, Chierchia et al. 2001, Papafragou and Musolino 2003, Guasti et al. |2005, Foppolo et al.| 2012, among many others). For example, Noveck (2001) presented underinformative sentences like 12 to a group of 8-year-old children, 10-year-old children, and adults. The participants were asked if they agreed with the sentences. Participants who derived the scalar inference in $\sqrt{13}$ were expected to disagree with the sentence.

(12) Some giraffes have long necks.

(13) Not all giraffes have long necks.

Noveck reported that child participants rejected the underinformative statements far less often than the adult participants did. This pattern of responses was taken as evidence that children derived fewer inferences than adults did. This result is representative of much of the subsequent work investigating children's performance on scalar inferences (though see Katsos and Bishop 2011 for evidence that features of the task, such as the number of response options provided to children, can influence their performance). It is worth noting, however, that many of the previous developmental studies have focused on a fairly restricted set of scalar inferences, namely those involving the scalar quantifiers 'some'/'all', the logical connectives 'or'/'and', and the modals 'may'/'must'. Importantly, the derivation of such scalar inferences involves alternatives generated through lexical replacement. More recent work has turned to investigating other inferences, including those derived from non-replacement alternatives.

\subsubsection{Adult-like behaviour}

In the recent formal semantics/pragmatics literature, a wide range of inferences have received a scalar inference-based analysis (Levinson 2000, Spector 2007, Fox 2007, Klinedinst 2007, Thomas 2012, Romoli 2013, Chierchia||2013). Subsequent studies investigating children's knowledge of these diverse inferences have produced some surprising results (Zhou et al. 2013, Tieu et al. 2014, 2016, 
Hochstein et al. 2016, Bill et al. 2016, Singh et al. 2016, Tieu et al. 2017), including the finding that some of these inferences are derived quite readily by young children.

One example of an inference that children have been reported to compute at adult-like rates is the free choice inference, investigated by Tieu et al. (2016). Tieu et al. investigated Mandarin-speaking 4-year-old children's interpretation of disjunction under deontic modals, as well as English-speaking 5-year-old children's interpretation of free choice 'any' under deontic modals. Both sentence types give rise to free choice inferences, which have received a scalar inference analysis in the literature (Kratzer and Shimoyama 2002, Alonso-Ovalle 2005. Fox 2007, Klinedinst 2007, Chemla 2009) 17 For example, a sentence like (14) gives rise to the free choice inference in (15).

(14) The elephant is allowed to catch a big butterfly or a small butterfly.

(15) The elephant is allowed to catch a big butterfly and the elephant is allowed to catch a small butterfly.

Tieu et al. (2016)'s results provided some preliminary evidence in support of the proposal that children are capable of computing inferences that can be generated using non-replacement alternatives, as compared to ones that require lexical replacement of alternatives. Tieu et al. used a Truth Value Judgment Task (Crain and Thornton 1998), in which the child participants were presented with free choice sentences (e.g., (14)), in contexts that were inconsistent with the free choice inference (e.g., (15)). That is, in the context, the character mentioned in the target sentence (e.g., the elephant) was only permitted to perform one of the mentioned actions (e.g., catch a big butterfly). The study compared children's performance with free choice inferences and standard scalar inferences involving plain disjunctions (i.e. the exclusivity inference). Tieu et al. found that the Mandarin-speaking children derived the exclusivity inference from test sentences with plain disjunction at a typically low rate $(18 \%)$, whereas they derived free choice inferences from sentences that contained a deontic modal verb at a significantly higher rate $(91 \%)$. Notably, both the free choice inference and the exclusivity inference conditions were tested within subjects, using the same Truth Value Judgment Task and similar materials, suggesting the divergent performance on the two inferences cannot be explained by differences in methodology 8

As another example, children have been reported to interpret numeral terms as exact (Papafragou and Musolino 2003, Barner and Bachrach 2010, Huang et al. 2013). On certain accounts, such an interpretation involves the derivation of a scalar inference (Horn 1972, Levinson 2000); for instance, 'two' is interpreted as 'exactly two' through the negation of the stronger alternatives 'three', 'four', etc.

\footnotetext{
${ }^{7}$ See Zimmermann (2000), Geurts (2005) and Barker (2010) for alternative analyses.

8 Tieu et al. (2016) report similarly high rates of free choice inferences from free choice English 'any', and Huang and Crain 2014 report similar rates of free choice inferences for Mandarin renhe 'any'.
} 
Turning to ad-hoc inferences, Stiller et al. (2015) presented 2- to 4-year-old children with sentence descriptions (e.g., My friend has glasses) and three corresponding pictures. The participants' task was to identify which of three faces the sentence was describing. The three faces created an ad-hoc scale (i.e $<$ face with no glasses, face with glasses, face with glasses and hat $>$ ). If participants derived the relevant ad-hoc inference for the target sentence description (i.e $m y$ friend does not have a hat), they were expected to select the face with glasses and no hat. Stiller et al. reported that 3-year-old children and 4-year-old children derived ad-hoc inferences approximately $75 \%$ of the time.

In yet another example of children's relative success, Hochstein et al. (2016) investigated 4- to 5-year-old children's derivation of ignorance inferences. These authors reported that 5-year-old children derived ignorance inferences at a rate of $70 \%$, while they computed exclusivity inferences only $30-40 \%$ of the time.

Finally, children have also been reported to succeed in accessing exhaustive interpretations similar to those in the derivation of scalar implicatures, when interpreting 'only'-sentences involving context-dependent scales (Barner et al. 2011). Barner et al. (2011) presented 4-year-old children with pictures depicting three objects/animals (e.g., cat, cow, dog), all of which were doing the same activity (e.g., sleeping). Children were asked one of two questions; Are only some of the animals sleeping? or Are only the cat and the cow sleeping? The former question is associated with context-independent alternatives, $<$ some...all $>$, whereas the latter is associated with context-dependent alternatives, $\langle$ cat, cow, $\operatorname{dog}>$ ). A negative answer to these questions was taken as evidence of an exhaustive interpretation, i.e. Only some, not all, of the animals are sleeping. Barner et al. reported that children accessed the exhaustive interpretation in the context-independent condition $33 \%$ of the time, whereas they did so in the context-dependent condition $86 \%$ of the time.

\subsubsection{Explaining children's variable behaviour}

The majority of previous studies investigating children's abilities with scalar inferences and exhaustive interpretations have reported low rates of derivation in children. However, some recent studies have revealed a series of inferences that children successfully compute. This disparity in findings calls for an explanation for children's variable success. The need for an explanation is especially pressing on unified accounts that treat all of the relevant inferences in the same way.

Two main kinds of explanations for the developmental findings have been proposed. On one account, children's difficulties with scalar inferences are attributed to limitations in their pragmatic knowledge (Noveck 2001, Katsos and Bishop 2011, Skordos and Papafragou 2016). This line is taken, for example, by Katsos and Bishop (2011), who propose that children are more 'pragmatically tolerant' than adults. On this view, even when children derive scalar inferences, they are nevertheless more likely than adults to accept a target sentence, despite the fact that the inference makes the sentence false. Similarly, Skordos and Papafragou (2016) propose that children have a limited understanding of which alternative sentences are relevant in a given context (see Noveck 2001 
for a similar idea). This limitation is expected to affect children's derivation of scalar inferences, since alternative sentences need to be perceived as 'relevant' before they can be negated.

Such pragmatic explanations are able to account for the typical observation of low rates of scalar inferences in children. However, it is unclear how such accounts can be extended to cases where children perform at adult-like rates in deriving inferences (e.g., free choice inferences). One would expect the proposed limitation ascribed to children (pragmatic tolerance or limited understanding of relevance) to have a uniform effect across different types of inferences, contra recent findings.

A second kind of explanation that attempts to account for children's selective success on scalar inferences is one we will broadly term the Alternatives-based approach. This approach attributes children's difficulties to a limitation in children's ability to generate alternatives. There are different proposals regarding the cause of this difficulty. Some explanations appeal to children's processing limitations, suggesting that the process of generating alternatives is costly, and therefore challenging for children (Chierchia et al. 2001, Gualmini et al. 2001, Reinhart 2006, Tieu et al. 2016). Others appeal to limitations in children's knowledge of lexical scales, suggesting that children often have not learned the scales required to generate alternatives through lexical replacement (Barner and Bachrach 2010, Barner et al. 2011). This difficulty with generating alternatives means that children will experience difficulties deriving the associated scalar inferences. In this way, the Alternatives-based approach, like the pragmaticbased explanations, can account for why children have been found to struggle with many scalar inferences.

In contrast to the pragmatic-based explanations, however, the Alternativesbased approach can also account for why children have been found to succeed on certain scalar inferences. Crucially, nearly all of the inferences that children have been found to compute successfully do not involve alternatives generated through lexical replacement. Instead, they involve non-replacement alternatives. That is, they involve alternatives generated through the simplification of the asserted sentence (or, in the case of ad hoc implicatures, through alternatives that are made salient and easily retrievable from the context). For example, as already mentioned, a sentence like (14) (repeated in (16)) is often associated with the free choice inference in (15) (repeated in (17)). Deriving free choice inferences as scalar inferences involves the alternative sentences in (18) and (19). However, these are non-replacement alternative sentences as they are generated by deleting parts of the assertion in (16), and not by lexical replacement of scalar terms (Kratzer and Shimoyama 2002, Alonso-Ovalle 2005, Fox 2007. Klinedinst 2007, Chemla 2009). As mentioned, Tieu et al. (2016) found that children readily derived the free choice inferences associated with these kinds of alternatives.

(16) The elephant is allowed to catch a big butterfly or a small butterfly.

(17) The elephant is allowed to catch a big butterfly and the elephant is allowed to catch a small butterfly. 
(18) The elephant is allowed to catch a big butterfly.

(19) The elephant is allowed to catch a small butterfly.

If we assume that, aside from the proposed limitation associated with the lexical replacement of alternatives, children are in all other relevant respects adult-like, then at least some version(s) of the Alternatives-based approach will predict children to readily derive scalar inferences like (17). For example, children's limited knowledge of lexical scales, as proposed by Barner and Bachrach (2010), will not affect their ability to compute scalar inferences that do not involve knowledge of lexical scales, namely those derived from non-replacement alternatives.

The predictions of proposals attributing children's difficulties to processing limitations (e.g., Reinhart 2006), are somewhat less clear (see Singh et al. 2016, footnote 32, for some considerations that may speak against a processingbased account). Such proposals could in fact be taken to predict that children's behaviour across all scalar implicatures should be uniform, assuming all implicatures are highly demanding in terms of processing resources. On the other hand, if we assume that alternatives derived through lexical replacement are more costly than alternatives generated through some other source (e.g., by simplifying the assertion), then these processing accounts will also predict that children should be more successful at deriving scalar inferences associated with non-replacement alternatives (see Chemla and Bott 2014). In any case, in the remainder of this paper, when we refer to the 'Alternatives-based approach', we are only referring to explanations that predict that children will more readily derive scalar inferences from non-replacement alternatives.

The next section illustrates how the predictions of the Alternatives-based approach can be tested further by focusing on other inferences derived from nonreplacement alternatives. Specifically, we will focus on testing the predictions of the Alternatives-based approach for sentences containing disjunction under a universal quantifier.

\subsection{Testing the Alternatives-based approach}

As noted earlier, a sentence like 20 is associated with the distributive inference in (21) and the conjunctive inference in 222. Crucially, as outlined in Appendix $B$ and $C$ the alternatives that are required to compute these inferences are not generated through lexical replacement 9 Rather, the relevant alternatives in (23) and 24) are non-replacement alternatives that can be generated by deleting parts of the asserted sentence in 20110

\footnotetext{
${ }^{9}$ Note that Crnič et al. (2015) argue against deriving the distributive inference through a traditional scalar inference process. For our purposes, what matters is that the process they argue for is the same with respect to the nature of the alternative sentences. The Alternatives-based approach therefore makes the same predictions regarding children's behaviour.

${ }^{10}$ Note that the derivation of the conjunctive inference from an assertion like 20 is only possible on certain theories of scalar implicature computation, namely theories that allow the latter to be embeddable (e.g., Fox 2007); see Appendix C for details and an example of derivation.
} 
(20) Every elephant caught a big butterfly or a small butterfly.

(21) Some elephant caught a big butterfly and some elephant caught a small butterfly.

(22) Every elephant caught both a big butterfly and a small butterfly.

(23) Every elephant caught a big butterfly.

(24) Every elephant caught a small butterfly.

The Alternatives-based approach, therefore, predicts that children should readily compute distributive inferences like (21) and conjunctive inferences like (22), in response to sentences like (20), since both kinds of inferences are derived through non-replacement alternatives $\left.{ }^{11}\right|^{2}$

Our predictions for adults, on the other hand, are different. While adults are expected to derive the distributive inference, they are not expected to derive the conjunctive inference. This is because adults, unlike children, can presumably generate the alternative involving lexical replacement (i.e. the alternative in which disjunction is replaced by conjunction), which is thought to 'block' the derivation of the conjunctive inference (see Fox 2007, Singh et al. 2016, and Tieu et al. 2017 for relevant discussion).

In sum, we are assuming that the distributive inference and the conjunctive inference are members of a natural class of scalar inferences, namely, inferences derived through non-replacement alternatives (Katzir 2007, Fox and Katzir 2011). Notably, for a sentence like (20), the distributive and the conjunctive inferences are the only inferences that can be derived via non-replacement alternatives (see Appendix $\mathrm{D}$ for details). Importantly for our purposes, the Alternatives-based approach predicts that children should be able to derive both kinds of inferences, since no lexical replacement is required. Since we do not expect adults to compute conjunctive inferences, we cannot expect that the two inferences will be computed at exactly the same rates in children and adults. However, the Alternatives-based approach does predict that children should compute this class of inferences (i.e. either the distributive or the conjunctive inference) at a similar rate to adults ${ }^{13}$ This prediction was investigated in the present experimental study by assessing the rates at which adults and children derive distributive and conjunctive inferences from sentences like 20 .

\footnotetext{
${ }^{11}$ As pointed out by an anonymous reviewer, the assumption that conjunctive inferences are a genuine possibility derived through non-replacement alternatives, as opposed to arising due to some general confusion on the part of children, is predicated on the proposal in Singh et al. (2016), who provide supporting evidence that children indeed access such conjunctive inferences.

${ }^{12}$ Note also that the conjunctive inference asymmetrically entails the distributive inference. This makes it difficult to determine whether or not the child participants derive both inferences simultaneously. We will return to this issue.

${ }^{13}$ Notice that this picture diverges from a more classic view on which children only access the 'basic', unenriched meaning of sentences like 20 (e.g., Noveck 2001). In the present case, the Alternatives-based approach actually predicts that not only will children derive the same inference as adults (i.e. the distributive inference), they may also derive inferences that adults do not (i.e. the conjunctive inference).
} 


\section{Experiment}

The present experiment was devised to determine the extent to which children derive distributive and/or conjunctive inferences from sentences in which disjunction is embedded under the universal quantifier. Both of these inferences rely on non-replacement alternatives. Therefore, the experimental hypothesis, based on the Alternatives-based approach, was that the child participants would compute such inferences at adult-like rates. This finding would be in contrast to the findings of much previous research on inferences that require lexical replacement of scalar terms. In these studies, the child participants were found to derive inferences at significantly lower rates than adult participants.

\subsection{Method}

\subsubsection{Participants}

Seventeen monolingual English-speaking adults (Macquarie University undergraduate students, all females) and 20 monolingual English-speaking children (4;01-5;08, M=4;05, 5 females, 15 males) participated in the experiment. The child participants had no history of language impairment or delay. The adults took part in the experiment for course credit, or for a payment of 15AUD. Children were recruited from several on-campus daycares at Macquarie University, and from a Macquarie University child research participant database. Informed consent was obtained from the adult participants, and from the parent/guardian of the child participants.

\subsubsection{Procedure}

The experiment used a version of the Truth Value Judgment Task (TVJT) (Crain and Thornton 1998). Participants listened to a series of short stories, illustrated with photographs of toy props, on a laptop computer. After each story, a puppet appeared on the computer screen to utter the target sentence. Participants were asked to judge whether the puppet's sentence was right or wrong. If the participant said that the puppet was wrong, they were asked to provide a justification ("Why?" or "What really happened in the story?"). Some children also spontaneously provided follow-up justifications for yes-responses. Adults were tested using the same materials, but were asked to provide written responses, including written justifications for yes- and no-responses.

Each child was tested individually, either in the lab or in a quiet room at

their preschool. Adult participants were tested simultaneously in groups of three. The two test conditions were conducted in two sessions, 7-9 days apart, each lasting approximately 15 minutes. This was done to separate the DISTRIBUTIVE and NON-DISTRIBUTIVE conditions, reducing the chance that participants' responses in one condition might influence their responses in the other condition. The order in which the two sessions were presented was counterbalanced across participants. 


\subsubsection{Materials}

Each participant was presented with four target sentences in the NON-DisTRIBUTIVE condition and four target sentences in the DistRIBUTIVE condition, as well as two clearly true and two clearly false control sentences. The control sentences included the universal quantifier every, without disjunction. In addition, four filler sentences were included, for a total of 12 trials. The sentences were presented by the puppet using pre-recorded videos.

\section{Test conditions}

Each trial consisted of a single story. In each story, there were three characters and two sets of objects. In each story, each of the characters considered the two sets of objects in front of them; they then made a decision to perform a pre-designated action on one of the two objects, and then proceeded to perform that action. Once all the characters had completed the action, the experimenter asked the puppet to describe what the characters had done in the story. The puppet then responded with the target sentence. To illustrate a typical trial, the target sentence 26 ) was associated with the story in 25$]$.

(25) Example of Distributive target story

This is a story about three elephants. The elephants have come to a park to catch butterflies. There are big butterflies and small butterflies. The big butterflies are hard to catch, but the small butterflies are easy to catch. Let's see which butterflies the elephants decide to catch. The first elephant says: "I am not very fast, so I will catch a small butterfly." So she catches a small butterfly. The second elephant says: "I am very fast, so I will catch a big butterfly." So he catches a big butterfly. The third elephant says: "I am also very fast, so I will also catch a big butterfly." So he catches a big butterfly. And here are the elephants, with the butterflies that they caught [see Figure 1].

Experimenter: Now let's see if $<$ Puppet's name $>$ was paying attention. $<$ Puppet's name $>$, what did the elephants do?

(26) Puppet: Every elephant caught a big butterfly or a small butterfly.

In the DisTRIBUTIVE condition, participants who computed the literal meaning of the sentences were expected to accept them, since every elephant caught a big butterfly or a small butterfly. Participants who computed the distributive inference were also expected to accept the test sentences, since at least one elephant caught a big butterfly and at least one elephant caught a small butterfly. However, participants who computed the conjunctive inference were expected to reject the target sentences, since none of the elephants caught both a big butterfly and a small butterfly.

In the NON-DISTRIBUTIVE condition, all of the characters acted upon the same kind of object (e.g., all of the elephants caught a big butterfly). As in the DISTRIBUTIVE condition, participants who computed the literal meaning were 


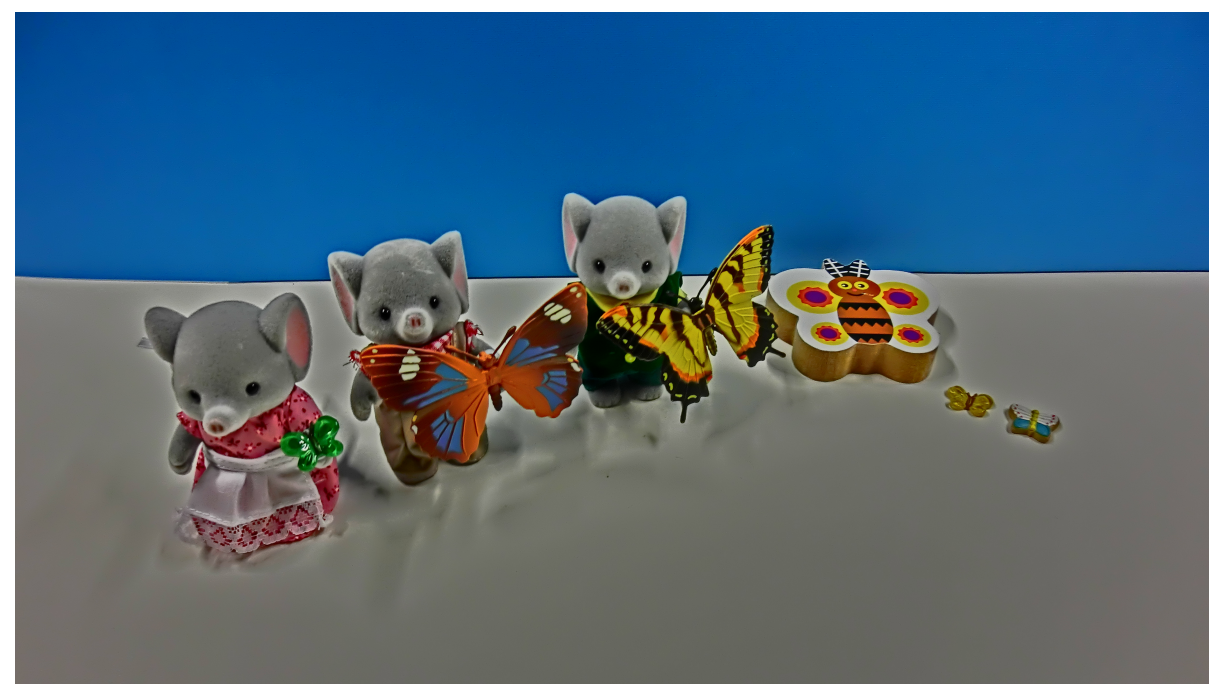

Figure 1: Final picture associated with the story in 25). Each elephant is holding the butterfly they caught.

expected to accept the target sentences. Participants who computed the conjunctive inference were again expected to reject the target sentences, since none of the elephants caught both a big butterfly and a small butterfly. Participants who computed the distributive inference were also expected to reject the target sentences, since none of the elephants caught a small butterfly. A summary of the expected pattern of responses in the two conditions is presented in Table 1 . Note that, as outlined in Appendix D, these response patterns are not expected on any other possible interpretations of our target sentences.

\begin{tabular}{l|c|c} 
& Distributive & Non-Distributive \\
\hline Literal interpretation & accept & accept \\
Distributive interpretation & accept & reject \\
Conjunctive interpretation & reject & reject
\end{tabular}

Table 1: Expected responses to the Distributive and Non-Distributive target sentences according to the three possible interpretations.

\section{Controls and fillers}

In addition to the eight target sentences, participants also received four control trials involving sentences containing the universal quantifier every but no disjunction. These control items were designed to ensure that participants understood the basic meaning of the universal quantifier. Participants received 
two clearly true and two clearly false controls. For example, on one false control item, three horses have to decide which of two vehicles to drive, a car or a boat. Two of the horses end up driving a boat, and one drives a car. The puppet then utters the false target in (27).

(27) Every horse drove a boat.

In addition to the targets and controls, participants also received four filler items, which could either be associated with a yes-response or a no-response. The experimenter determined which judgment to elicit from the participant, depending on the number of yes-responses and no-responses the participant had produced on previous trials. In this way, the experimenter ensured that each participant produced an equal number of yes-responses and no-responses.

\subsection{Results}

Participants had to correctly answer at least $75 \%$ of the filler trials and at least $75 \%$ of the control trials in order to be included in the analysis. All participants passed this criterion, and no participants were excluded from the analysis.

The performance by child and adult participants in the target conditions is presented in Figure 2, plotted as the proportion of target sentence rejections in the Distributive and Non-Distributive conditions.

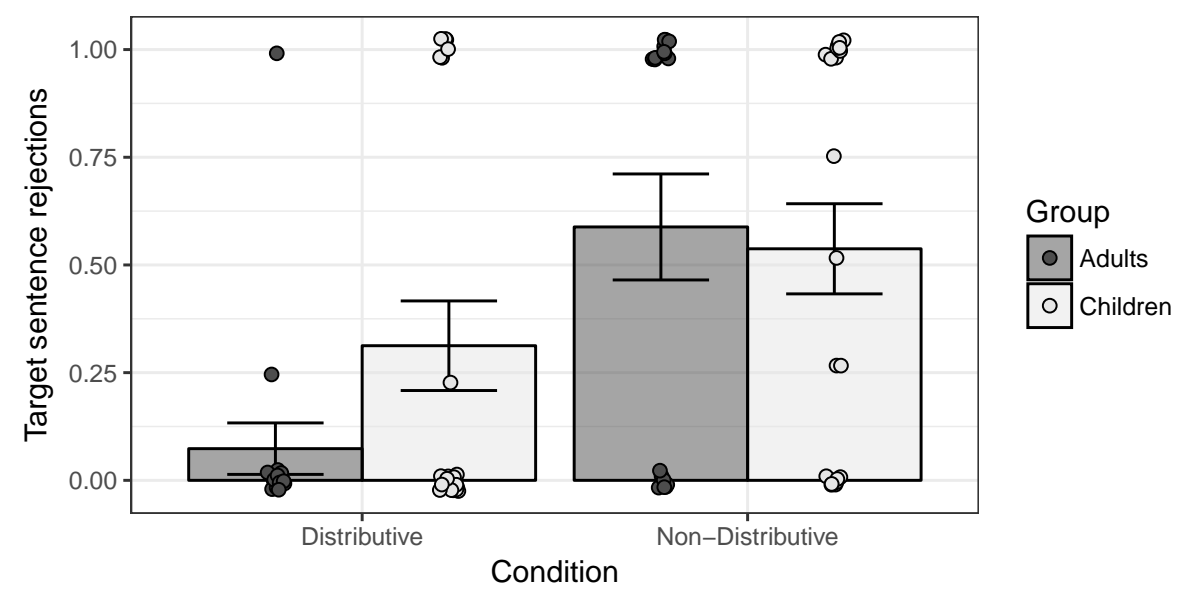

Figure 2: Proportion of target sentence rejections by Group and Condition. Each dot represents an individual participant's mean rejection of target sentences in that condition. A horizontal jitter of .1 and vertical jitter of .025 were applied to the dots for easier visualisation.

The data were analysed using Generalized Mixed-Models (GLMER) in R (version 3.3.3) with the LanguageR package (version 1.4.1). In the analysis, we used a backward elimination procedure to compare the goodness-of-fit of the 


\begin{tabular}{l|c|c|c|c} 
Predictor & Est. & SE & Z Wald & $\boldsymbol{p}$ \\
\hline (Intercept) & 10.74 & $2 . .29$ & 4.70 & $<.001$ \\
Condition - Non-Distributive & -20.02 & 3.11 & -6.43 & $<.001$ \\
\hline
\end{tabular}

Summary of fixed effects in the logit model $(N=296$; log-likelihood $=-68.2)$.

Table 2: The best fitting model of the data. Adult and Distributive were used as reference levels (0) for Group and Condition factors, respectively.

models. We started with the maximal structure that allowed the models to converge, following the recommendation by Barr (2013). This maximal model contained two random intercepts (for participants and for items) and an individual adjustment of the condition for each participant (random slope). Group (Adult vs. Child), Condition (Distributive vs. Non-Distributive), and the interaction of Group-by-Condition were included as fixed effects. The reference categories in our analysis were 'Adult' for Group and 'DistRIBUTIVE' for Condition.

We evaluated whether each of the predictors (i.e. fixed effects) significantly contributed to the model's fit by comparing a model including that predictor against another that did not include it, using a $\chi$-square test (Jaeger 2008). Subsequently, we calculated the $z$-value, based on the Wald statistic, which allows for an estimation of the statistical significance of each predictor included in the model.

The fixed effect of Condition made a significant contribution to the fit of the model $\left(\chi^{2}(1)=12.67, p<.001\right)$, whereas the interaction of the two fixed effects $\left(\chi^{2}(1)=.38, p=.54\right)$ and the fixed effect of Group $\left(\chi^{2}(1)=.01, p=.91\right) \mathrm{did}$ not contribute significantly to the fit of the model.

Estimated coefficients, their standard errors, Z-values, and associated pvalues for the chosen model are reported in Table 2.

The results reveal that target sentences in the Non-Distributive condition were rejected at a significantly higher rate than target sentences in the Distributive condition. No difference in target sentence rejection was found between the two groups.

Based on the majority of judgments produced by each individual participant in each condition, we categorised the participants into different responder types, i.e. "Literal", "Distributive", and "Conjunctive". A summary of the responder types is presented in Table 3. For example, a participant was classified as "Distributive" if they accepted at least three of four target sentences in the DisTRIBUTIVE condition and rejected at least three of four target sentences in the Non-Distributive condition. 


\begin{tabular}{l|c|c} 
& Distributive & Non-Distributive \\
\hline Literal responder & accept & accept \\
Distributive responder & accept & reject \\
Conjunctive responder & reject & reject
\end{tabular}

Table 3: Expected response patterns for the Distributive and NonDISTRIBUTIVE targets, by category of responder.

Three children were excluded from the categorisation, as one gave mixed responses, and two produced a pattern of responses that was not predicted by any of the relevant interpretations. The remainder of the participants fell into one of the three categories. Table 4 presents the number of participants in each category.

\begin{tabular}{l|c|c} 
& Child & Adult \\
\hline Literal responders & 7 & 7 \\
Distributive responders & 7 & 9 \\
Conjunctive responders & 3 & 1
\end{tabular}

Table 4: Distribution of responder types across the two groups.

Since our primary interest is in identifying whether children would derive an inference from the target sentences (whether distributive or conjunctive), Table 5 collapses the Distributive and Conjunctive responders into a single category of 'Inference' responders; these are participants who computed one inference or the other.

\begin{tabular}{l|c|c} 
& Child & Adult \\
\hline Literal responders & 7 & 7 \\
Inference responders & 10 & 10
\end{tabular}

Table 5: Distribution of responder types across the two groups, collapsing Distributive and Conjunctive responders.

As can be seen in Table 5 , children and adults patterned identically in terms of the distribution of literal responders and inference responders. Table 5 shows that children were as likely as adults to compute literal meanings, and as likely to compute inferences.

\section{Justifications}

In addition to yes-/no-responses, we also collected justifications from each participant, which were generally consistent with their categorisation as a Literal, 
Distributive, or Conjunctive responder. For instance, the justifications from the 7 children and 7 adults who gave literal responses (accepting both the DISTRIBUTIVE and NON-DISTRIBUTIVE targets) generally focused on repeating the basic elements of the relevant story, e.g. (Yes) every girl ate a big or a small pizza; (Yes) two dogs drove a big car and one dog drove a small car; (Yes) every cow washed a big dish.

The 7 children and 9 adults who were categorised as Distributive responders accepted the DISTRIBUTIVE targets. The adult participants justified their yes-responses by recounting what had happened in the story, e.g. (Yes) every elephant did catch either a big or small butterfly; (Yes) two girls ate a big pizza and one ate a small pizza. The Distributive responders also rejected the NONDISTRIBUTIVE targets, justifying their no-responses by pointing out that all of the characters had (only) acted on the one set of items, e.g. (No) all the fairies ate a big cookie, (No) they all did wash a big dish.

Finally, the 3 children and 1 adult who were categorised as Conjunctive responders rejected the Distributive targets, justifying their no-responses by recounting what had happened in the story, e.g. (No) because the two girls said the big pizza and one said the small one; (No) two have big teas, and one got a little tea. The Conjunctive responders also rejected the Non-DISTRIBUTIVE targets, explaining that the characters had only acted on one of the sets of items, e.g., (No) everyone opened a big can. Her said everyone opened a big one and a small one; (No) all of them drank the big ones.

In sum, participants' justifications were generally consistent with the interpretations attributed to them.

\section{Discussion}

The aim of this experiment was to investigate the predictions of the Alternatives-based approach (Chierchia et al. 2001, Gualmini et al. 2001, Reinhart 2006, Barner and Bachrach 2010,|Barner et al.|2011, Tieu et al. 2016). Specifically, we aimed to test the prediction that children would be able to compute inferences that were derived from non-replacement alternatives, that is, from alternatives that could be generated from the asserted sentences through deletion.

We did this by investigating adults' and children's interpretations of sentences like (28). Specifically, we looked at whether their interpretations included distributive inferences such as (29) (Crnič et al. 2015) or conjunctive inferences such as (30) (Singh et al. 2016), as both these inferences have been proposed to be derived as scalar inferences from non-replacement alternatives. The Alternatives-based approach would lead us to expect children to readily access (i.e. at a similar rate as adults) an inference-based reading, be it from a distributive inference or a conjunctive inference.

(28) Every elephant caught a big butterfly or a small butterfly.

(29) Some elephant caught a big butterfly and some elephant caught a small butterfly. 
(30) Every elephant caught both a big butterfly and a small butterfly.

Our experimental results indicate that children indeed accessed inference-based readings of the test sentences, and importantly, we did not find any difference between the rates at which children and adults accessed these readings, in line with the prediction of the Alternatives-based approach. Specifically, both groups exhibited the same decrease in target sentence acceptance when the sentences were used to describe contexts that were inconsistent with both the distributive and conjunctive inferences. This indicates that children were deriving at least one of the inferences of interest, as readily as adults did, consistent with the predictions of the Alternatives-based approach 14

Children's rejections in the Distributive condition, in which the target sentences were presented in the contexts that were consistent with the distributive inference but not with the conjunctive inference, indicate that children were indeed computing the conjunctive inference to some extent. The results suggest that children's rejections in the NON-DisTRIBUTIVE condition were likely driven by a mix of distributive and conjunctive inferences. This is because if children were only deriving conjunctive inferences, we would not expect a difference in their behaviour between the two conditions (as both conditions were incompatible with the conjunctive inference). The individual participant data were also informative, indicating the presence of each of the relevant readings (literal, distributive, and conjunctive). Importantly, we found no differences in how children and adults were distributed across the Literal and Inference categories. In sum, the results of our experiment are consistent with the prediction of the Alternatives-based approach: when a scalar inference is derived from non-replacement alternatives, children can derive it readily.

Focusing on the conjunctive inference, we observed evidence for this inference in the responses of three children and one adult. The responses by the children were consistent with those of children in previous studies by Singh et al. (2016) and Tieu et al. (2017). However, the conjunctive inference was far less robust in the present experiment, as compared to the findings reported in the previous studies. It is worth noting in this regard that, although our experiment included a condition that was consistent with the distributive inference, there was no condition that was consistent with the conjunctive inference. If children derived both inferences, they may have perceived the sentence to be ambiguous between a distributive and a conjunctive interpretation. If so, then the fact that the distributive inference was consistent with one of the experimental conditions may have weighed in its favour, leading participants to resolve the ambiguity by computing the distributive inference. A future study might include, in addition to our conditions, a condition in which the conjunctive inference is made true.

\footnotetext{
${ }^{14}$ An anonymous reviewer points out that some of the adult and child rejections may arise due to a pragmatic oddness (i.e. oddness due to something other than falsehood); for instance, the reviewer observes that in the NON-DisTRIBUTIVE condition, it is odd to utter "every $\mathrm{Y}$ is A or B" when "every $\mathrm{Y}$ is A" would suffice. It is somewhat difficult in the present study to evaluate the possible role of oddness. As the reviewer notes, however, a future study might make use of a three-valued task such as the one presented in Katsos and Bishop (2011), which might reveal more fine-grained differences in types of 'rejection'.
} 
To sum up, the results of this study reveal that children successfully derive distributive and conjunctive inferences, which are both scalar inferences derived from non-replacement alternatives. This finding contrasts with previous findings showing that children struggle to derive scalar inferences involving alternatives generated through lexical replacement (e.g., Noveck 2001, Chierchia et al. 2001, Papafragou and Musolino 2003, Guasti et al. 2005, Foppolo et al. 2012 among many others). Of course, our results need to be interpreted with a certain level of caution, as we did not provide a direct comparison of scalar inferences derived from replacement alternatives and those derived from nonreplacement alternatives. A future study could directly compare the derivation of non-replacement inferences (e.g., distributive/conjunctive inferences) and inferences based on lexical replacement. This would provide a more explicit test of the prediction that children will be uniquely successful in deriving scalar inferences from non-replacement alternatives.

\subsection{Quantifier spreading}

An anonymous reviewer noted that the response pattern we associated with 'conjunctive responders' (i.e. children who rejected target sentences in both the Distributive and the Non-Distributive conditions) might also be attributable to a well-known phenomenon called 'quantifier spreading' (Philip 1991, 1995). This is a phenomenon whereby children are observed to reject sentence descriptions headed by a universal quantifier in situations where there are 'leftover' objects that are not matched with an agent. For example, Philip (1995) found that children would often reject a sentence like (31) if it were used to describe a context in which every man was riding a donkey, but there was a donkey that was not being ridden.

(31) Every man is riding a donkey.

In both test conditions in our experiment, there were objects/animals (e.g., butterflies) that were not matched with an agent. Therefore, as the anonymous reviewer notes, if children accessed a quantifier spreading interpretation, they would be expected to reject the target sentence in both test conditions. While we are not able to completely rule out the possibility that these children's responses were based on a quantifier spreading interpretation, we believe it is implausible for the following reasons.

First, the justifications provided by the relevant children are not in line with what would be expected if they were accessing a quantifier spreading interpretation. If children accessed such an interpretation, we would expect their justifications to focus on the leftover objects, and the fact that they were not being acted upon. However, as we identified in Section 2.2. the justifications of the children providing a 'conjunctive response' tended to focus on the items that the characters had acted upon (e.g., two have big teas, and one got a little tea; see also Appendix E for the complete list of justifications given by children who gave no-responses). 
Second, a study by Sugisaki and Isobe (2001) found that when there was more than one 'leftover' object in the context, children's quantifier spreading interpretations reduced from $62.5 \%$ to $12.5 \%$ (see also Rakhlin 2007 for similar discussion). In our experimental conditions, there were always three leftover objects. Given Sugisaki and Isobe (2001)'s findings, it is unlikely that many (if any) of our child participant's responses were based on having accessed a quantifier spreading interpretation.

In sum, we believe that it is unlikely that the children's conjunctive response patterns were based on having accessed a quantifier spreading interpretation.

\subsection{The egalitarian interpretation}

Two of the child participants (who were included in the general data analysis but excluded from the individual categorisation) provided a response pattern that was not expected on any of the readings we identified. Instead, these participants appeared to access what might be called an egalitarian interpretation of the target sentences, meaning they would only accept the target sentence if each character had acted upon the same kind of object. That is, these two children rejected the target sentences in the DistRIBUTIVE condition and accepted them in the Non-Distributive condition. The justifications these child participants offered for rejecting the target sentences in the DisTRIBUTIVE condition indicated that they accepted the target sentences only if all of the characters performed the same action, e.g., (No, because) only one got a small butterfly; (No, because) one drank a small tea, two drank a big tea.

A similar pattern of responses was previously reported in a study by Boster and Crain (1993) and in a study by Kiguchi and Thornton (2016). Boster et al. tested 4-year-old children using sentences with disjunction, which were embedded under the universal quantifier, every, as in the present study. The authors of both studies report that a subset of their child participants accepted the test sentences only if all of the relevant characters performed the same action. Boster et al. proposed that across languages, children initially adopt this egalitarian interpretation to ensure that other interpretations can be acquired on the basis of positive evidence. This proposal is based on the observation that the egalitarian interpretation makes sentences true in a narrow range of circumstances, making it the 'subset' interpretation. Speakers of a number of languages judge sentences to be true in circumstances corresponding to the egalitarian interpretation (e.g., Mandarin Chinese (An 2015)).

We should note that, while striking, the presence of this response pattern does not bear directly on the interpretations we have attached to the other response patterns produced by participants. That is, the egalitarian interpretation appears to be a distinct interpretation, unrelated to the target inferences. 


\section{Conclusion}

Recent developmental work has led to proposals that children's variable success in computing scalar inferences can be explained by appealing to the nature of the alternatives involved; we refer to this family of approaches as the Alternativesbased approach. This approach was the source of the specific prediction we investigated - namely that adults and children would derive scalar inferences based on non-replacement alternatives to a similar extent. To test this prediction, we used sentences in which disjunction was embedded under a universal quantifier, e.g., every elephant caught a big butterfly or a small butterfly. Such sentences give rise to the distributive inference that some elephants caught a big butterfly and some elephants caught a small butterfly, and for children also to the conjunctive inference that every elephant caught a big butterfly and a small butterfly. Crucially, these inferences can be derived using non-replacement alternatives that can be formulated by simplifying the asserted sentence through deletion; as such, they do not rely on lexical replacement of alternatives. The findings of the present study reveal that children derive these scalar inferences at an adult-like rate. This result is in line with the predictions of the Alternativesbased approach, and provide support for it as a viable explanation of children's variable success in computing scalar inferences.

\section{Acknowledgements}

We would like to thank all the children and families who participated in the study. The research leading to these results was supported by the Australian Research Council Centre of Excellence in Cognition and its Disorders (CE110001021), the European Research Council under the European Union's Seventh Framework Programme (FP/2007-2013) / ERC Grant Agreement n.313610, ANR10-IDEX-0001-02 PSL*, ANR-10-LABX-0087 IEC, and the Leverhulme Trust, grant RPG-2016-100. No financial interest or benefit arises from direct application of this research.

\section{Author Contributions}

C.B, E.P. and S.C. conceived the project; C.B. and E.P. designed the experiment in consultation with J.R., L.T. and S.C; C.B. and E.P. collected the data; E.P. analysed the data; E.P. and C.B. prepared the initial manuscript, J.R., L.T. and S.C. revised the manuscript.

\section{Conflict of interest}

The authors declare that no competing interests exist. 


\section{Supplementary material}

The data for this experiment are available online at https://semanticsarchive. net/Archive/mEOYjdkN/Pagliarini-Bill-Romoli-Tieu-Crain-AcqDistributiveInferences. html.

\section{Appendix: Deriving the distributive and conjunc- tive inferences}

In this appendix, we sketch a possible derivation of scalar inferences like the exclusivity inference (33), from the asserted sentence (32). Following this we will outline how the distributive inference and the conjunctive inference can be accounted for through this same scalar inference process.

(32) The elephant caught a big butterfly or a small butterfly.

(33) It's not the case that the elephant caught both a big butterfly and a small butterfly.

As mentioned, there is ongoing debate regarding the exact mechanism underlying scalar inferences. The main contribution of our study does not hinge on assuming any one particular account, but for the purposes of illustration, we will adopt a grammatical account of scalar implicatures (Chierchia 2006, Fox 2007). We will first sketch the main ingredients of this account and then show how it can derive the three inferences in turn.

\section{A Deriving the exclusivity inference}

The gist of the grammatical account is that scalar implicatures arise due to the presence of a covert exhaustivity operator exh, akin to 'only,' which quantifies over a sentence and its relevant alternatives. Intuitively, what exh generally does is strengthen the sentence as much as possible, while avoiding contradictions and arbitrary choices among the sentence's alternatives. The definition of exh is provided in (34), and the definition of the alternatives that can be negated, the 'excludable' alternatives, is provided in (35). In essence, exh takes a sentence and a set of alternatives as its input and outputs the conjunction of that sentence and the negation of a subset of its alternatives. An alternative is excludable if its negation doesn't contradict the literal meaning of the asserted sentence, and doesn't force us to accept any other alternative in the set 15

$$
\begin{aligned}
& \llbracket \operatorname{exh} \phi \rrbracket(w)=\llbracket \phi \rrbracket(w) \wedge \forall \psi \in \operatorname{ExCL}(\phi, A L T(\phi))[\neg \llbracket \psi \rrbracket(w)] \\
& \operatorname{ExCL}(\phi, X)= \\
& \{\psi \in X: \llbracket \phi \rrbracket \nsubseteq \llbracket \psi \rrbracket \wedge \neg \exists \chi[\chi \in X \wedge(\llbracket \phi \rrbracket \wedge \neg \llbracket \psi \rrbracket) \subseteq \llbracket \chi \rrbracket]\}
\end{aligned}
$$

\footnotetext{
${ }^{15}$ This is what Fox (2007) refers to as 'innocent exclusion.' Fox (2007) further refines the version above, but it will suffice for our purposes; see Fox (2007) for discussion.
} 
In the following, we turn to show how the ingredients above can derive the exclusivity inference, and, subsequently, the distributive and conjunctive inferences. Let us return to the sentence in (36) and its inference, schematised as ' $(\mathrm{A} \vee \mathrm{B})$ '.

(36) The elephant caught a big butterfly or a small butterfly. $\rightsquigarrow$ The elephant didn't catch both a big and a small butterfly

The exclusivity inference is derived by assuming that exh is now underlyingly present in the sentence above as in (38) and that it quantifies over the alternatives in $37{ }^{16}$

$$
\begin{aligned}
& \text { Alt }=\{A \vee B, A, B, A \wedge B\} \\
& \operatorname{exh}[A \vee B]=(A \vee B) \wedge \neg(A \wedge B)
\end{aligned}
$$

As is easy to see, only the conjunctive alternative is excludable in this case, and the meaning obtained is the desired exclusivity inference $\neg(\mathrm{A} \wedge \mathrm{B})$, i.e. the elephant didn't catch both a big and a small butterfly ${ }^{17}$

\section{B Deriving the distributive inference}

In this subsection, we show that the ingredients above applied to a sentence like (39), schematised as ' $\mathrm{EVERY}_{x}(\mathrm{~A} x \vee \mathrm{B} x)^{\prime}$ ', can derive the distributive inference as well.

(39) Every elephant caught a big butterfly or a small butterfly. $\rightsquigarrow$ Some elephant caught a big butterfly and some elephant caught a small butterfly

We are assuming the set of alternatives in 400; the derivation is provided in (41). The crucial bit of the outcome that corresponds to the distributive inference is the negation of the alternatives corresponding to the disjuncts: every elephant caught a big butterfly or a small butterfly, and not every elephant caught a big butterfly and not every elephant caught a small butterfly, therefore it must be that some elephants caught a big butterfly and some elephants caught a small one.

$$
\operatorname{Alt}=\left\{\begin{array}{l}
\operatorname{EVERY}_{x}(\mathrm{~A} x \vee \mathrm{B} x), \\
\operatorname{EVERY}_{x}(\mathrm{~A} x) \\
\operatorname{EVERY}_{x}(\mathrm{~B} x) \\
\operatorname{EVERY}_{x}(\mathrm{~A} x \wedge \mathrm{B} x)
\end{array}\right\}
$$

\footnotetext{
${ }^{16}$ A key question for the grammatical account and for approaches to scalar implicatures in general, is how to determine the alternatives, which exh quantifies over. This is a very controversial topic in literature, which goes well beyond the scope of this paper. We will simply assume the alternatives that we need for the derivations below; see Breheny et al. (2016) and references therein for discussion.

${ }^{17}$ Notice that if we were to try and also exclude the single disjuncts, we would either obtain a contradiction with the assertion (by excluding both), or we would have to arbitrarily choose one of the two, and this is precisely what exh is designed not to do.
} 
(41) $\operatorname{exh}\left[\operatorname{EVERY}_{x}(\mathrm{~A} x \vee \mathrm{B} x)\right]=$ $\operatorname{EVERY}_{x}(\mathrm{~A} x \vee \mathrm{B} x) \wedge \neg \operatorname{EVERY}_{x}(\mathrm{~A} x) \wedge \neg \operatorname{EVERY}_{x}(\mathrm{~B} x) \wedge \neg \operatorname{EVERY}_{x}(\mathrm{~A} x \wedge \mathrm{B} x)=$ $\operatorname{EVERY}_{x}(\mathrm{~A} x \vee \mathrm{B} x) \wedge \operatorname{SOME}_{x}(\mathrm{~A} x) \wedge \operatorname{SOME}_{x}(\mathrm{~B} x) \wedge \neg \operatorname{EVERY}_{x}(\mathrm{~A} x \wedge \mathrm{B} x)$

In this way, it is possible for the distributive inference to be analysed as a scalar inference, derived as above ${ }^{18}$

\section{Deriving the conjunctive inference}

We turn now to outline how the conjunctive inference can be derived as a scalar inference, from an asserted sentence like 42 .

(42) Every elephant caught a big butterfly or a small butterfly. $\rightsquigarrow$ Every elephant caught a big butterfly and a small butterfly

There are three things that we need to mention in this respect. First, in order to derive the conjunctive inference from a sentence like $(42)$ via the scalar inference process, we need to compute the process at an embedded level. This is natural in the grammatical account we have been using above, where exh can appear embedded within a sentence. Second, it needs to be assumed that the scalar inference process can be applied recursively, again something that is natural in this account, by simply assuming more than one exh in the sentence. Finally, it needs to be assumed that, unlike adults, children do not generate the conjunctive alternative for a disjunctive sentence ${ }^{19}$ In fact, this is why the conjunctive inference is proposed to be a possible inference of disjunction only for children; if the conjunctive alternative were available, like it is assumed to be for adults, the conjunctive inference derivation would be blocked 20 Singh et al. (2016) justify this assumption by proposing that children are limited in their generation of alternative sentences (along the lines proposed by the Alternatives-based approach); in particular, children are proposed to experience difficulties generating alternative sentences involving lexical replacement, such as the conjunctive alternative.

The derivation is as follows: We assume that 42 is analysed as in (44), where not one but two exh appear in the scope of the quantifier ${ }^{21}$

$$
\operatorname{EVERY}_{x}\left(\operatorname{exh}_{1}\left(\operatorname{exh}_{2}(A x \vee B x)\right)\right)
$$

\footnotetext{
${ }^{18}$ As mentioned in fn.9, Crnič et al. $(2015)$ argue against deriving the distributive inference through this traditional scalar inference process. Their derivation is the same with respect to the nature of the alternative sentences. We therefore do not go into the details of their proposal here, however see Appendix D for the derivation they use.

${ }^{19}$ See Singh et al. (2016) for further discussion of this point.

${ }^{20}$ See Davidson (2013) and Bowler (2014) for evidence of conjunctive inferences of disjunction in adults in American Sign Language and Warlpiri.

${ }^{21}$ More precisely, we would need to assume an underlying logical form along the lines of 43 :

(43) $\operatorname{EVery}_{x}(\operatorname{ElePhant}(x))\left(\lambda x\left[\operatorname{exh}_{1}\left(\operatorname{exh}_{2}(A x \vee B x)\right)\right]\right)$
} 
We are assuming that the alternatives for exh $_{2}$ are those in (45), not including the conjunctive alternative, as mentioned above. As is easy to see, exh doesn't add anything to the sentence directly, as none of the alternatives in (45) is excludable. However, it plays a crucial indirect role by exhaustifying the alternatives for exh $h_{1}$, which are those in (46). When exh $h_{1}$ quantifies over these latter alternatives, the two alternatives corresponding to the two exhaustified disjuncts are excludable, so they end up being negated. Their negation, in conjunction with the assertion, gives rise to the conjunctive meaning shown in (47). When this meaning is composed with the rest of the sentence, we obtain (48), which corresponds to the desired conjunctive inference above that every elephant caught a big and a small butterfly.

$$
\begin{aligned}
& \mathrm{Alt}_{2}=\{(\mathrm{A} x \vee \mathrm{B} x), \mathrm{A} x, \mathrm{~B} x\} \\
& \mathrm{Alt}_{1}=\left\{\begin{array}{l}
\operatorname{exh}_{2}(\mathrm{~A} x \vee \mathrm{B} x)=(\mathrm{A} x \vee \mathrm{B} x) \\
\operatorname{exh}_{2}(\mathrm{~A} x)=(\mathrm{A} x \wedge \neg \mathrm{B} x) \\
\operatorname{exh}_{2}(\mathrm{~B} x)=(\mathrm{B} x \wedge \neg \mathrm{A} x)
\end{array}\right\} \\
& \operatorname{exh}_{1}\left(\operatorname{exh}_{2}(A x \vee B x)\right)= \\
& (A x \vee B x) \wedge \neg(\mathrm{A} x \wedge \neg \mathrm{B} x) \wedge \neg(\mathrm{B} x \wedge \neg \mathrm{A} x)= \\
& (A x \vee B x) \wedge(\mathrm{A} x \rightarrow \mathrm{B} x) \wedge(\mathrm{B} x \rightarrow \mathrm{A} x)= \\
& (A x \wedge B x) \\
& \text { (48) } \operatorname{EVERY}_{x}(A x \wedge B x)
\end{aligned}
$$

\section{No other inference explains the results}

In this appendix, we investigate a variety of possible parses for sentences of the form 'EVERY $x(A x \vee B x)$ ' involving one or more exh at different scope sites. We show that none of them are associated with an inference other than the conjunctive and distributive inferences, hence the pattern observed in our study (see also Singh et al. 2016 for analogous derivations). In essence, all of the parses explored below result either in the distributive inference, the conjunctive inference, no inference at all, or the two exclusivity inferences in (49) and (50).

$$
\begin{aligned}
& \neg \operatorname{EVERY}_{x}(A x \wedge B x) \\
& \operatorname{EVERY}_{x} \neg(A x \wedge B x)
\end{aligned}
$$

All of our target conditions were designed to be compatible with both of these inferences i.e. these inferences were always true in the test conditions, so they cannot account for the pattern of rejections we observed. We therefore conclude that the conjunctive and distributive inferences are what most plausibly drove the behavior displayed by our adult and child participants.

In the following, we sketch the derivations of the various parses in detail. We do this in two steps: we first consider scenarios where children cannot access the lexical conjunctive alternative, following the Alternatives-based approach (and Singh et al. 2016), and we then consider derivations that are possible when the conjunctive alternative is assumed to be available. 


\section{D.1 Parses based on non-replacement alternatives}

Let us return to a sentence of the form ' $\operatorname{EVERY}_{x}(A x \vee B x)$ ' and explore a variety of parses with exh at different sites, by keeping, for now, the assumption that children can only access non-replacement alternatives.

- With only one exh at the global level, we derive the distributive inferences (as already demonstrated in Appendix B).

$$
\begin{aligned}
& \operatorname{exh}\left(\operatorname{EVERY}_{x}(A x \vee B x)\right)= \\
& \operatorname{EVERY}_{x}(A x \vee B x) \wedge \neg \operatorname{EVERY}_{x}(A x) \wedge \neg \operatorname{EVERY}_{x}(B x)= \\
& \operatorname{EVERY}_{x}(A x \vee B x) \wedge \operatorname{SOME}_{x}(A x) \wedge \operatorname{SOME}_{x}(B x) \\
& \text { Alt }=\left\{\begin{array}{l}
\operatorname{EVERY}_{x}(A x \vee B x) \\
\operatorname{EVERY}_{x}(A x) \\
\operatorname{EVERY}_{x}(B x)
\end{array}\right\}
\end{aligned}
$$

- Having two global exh's lead again to the distributive inferences, i.e. the second exh does not add anything.

$$
\begin{aligned}
& \operatorname{exh}_{2}\left(\operatorname{exh}_{1}\left(\operatorname{EVERY}_{x}(A x \vee B x)\right)\right)= \\
& \operatorname{EVERY}_{x}(A x \vee B x) \wedge \neg \operatorname{EVERY}_{x}(A x) \wedge \neg \operatorname{EVERY}_{x}(B x) \wedge \neg\left(\operatorname{EVERY}_{x}(A x) \wedge\right. \\
& \neg \operatorname{EVERY}_{x}(B x) \wedge \neg\left(\operatorname{EVERY}_{x}(B x) \wedge \neg \operatorname{EVERY}_{x}(A x)\right)= \\
& \operatorname{EVERY}_{x}(A x \vee B x) \wedge \neg \operatorname{EVERY}_{x}(A x) \wedge \neg \operatorname{EVREY}_{x}(B x) \wedge \operatorname{EVERY}_{x}(A x) \leftrightarrow \\
& \operatorname{EVERY}_{x}(B x)= \\
& \operatorname{EVERY}_{x}(A x \vee B x) \wedge \neg \operatorname{EVERY}_{x}(A x) \wedge \neg \operatorname{EVERY}_{x}(B x) \\
& \operatorname{Alt}_{1}=\left\{\begin{array}{l}
\operatorname{EVERY}_{x}(A x \vee B x), \\
\operatorname{EVERY}_{x}(A x), \\
\operatorname{EVERY}_{x}(B x)
\end{array}\right\} \\
& \operatorname{Alt}_{2}=\left\{\begin{array}{l}
\operatorname{exh}_{1}\left(\operatorname{EVERY}_{x}(A x \vee B x)\right)=\operatorname{EVERY}_{x}(A x \vee B x) \wedge \neg \operatorname{EVERY}_{x}(A x) \wedge \neg \operatorname{EVERY}_{x}(B x), \\
\operatorname{exh}_{1}\left(\operatorname{EVERY}_{x}(A x)\right)=\operatorname{EVERY}_{x}(A x) \wedge \neg \operatorname{EVERY}_{x}(B x),
\end{array}\right\}
\end{aligned}
$$

- Having one local exh leads to no inference at all, as no alternative is excludable.

$$
\begin{aligned}
& \operatorname{EVERY}_{x}[\operatorname{exh}[A x \vee B x]]=\operatorname{EVERY}_{x}[A x \vee B x] \\
& \text { Alt }=\{(A x \vee B x), A x, B x\}
\end{aligned}
$$

- Having two local exh's leads us to the conjunctive inference (as demonstrated in Appendix C).

(58) $\operatorname{EVERY}_{x}\left[\operatorname{exh}_{2}\left[\operatorname{exh}_{1}[A x \vee B x]\right]\right]=$ $\operatorname{EVERY}_{x}[(A x \vee B x) \wedge(A x \leftrightarrow B x)]=$ $\operatorname{EVERY}_{x}[A x \wedge B x]$ 
(59) $\quad \mathrm{Alt}_{1}=\{(A x \vee B x), A x, B x\}$

(60) $\quad \mathrm{Alt}_{2}=\{(A x \vee B x), A x \wedge \neg B x, B x \wedge \neg A x\}$

- With one local exh and one global exh, we derive the distributive inferences (this is analogous to the derivation proposed by Crnič et al. (2015) for distributive inferences in adults).

$$
\begin{array}{ll}
\text { (61) } & \operatorname{exh}_{2}\left(\operatorname{EVERY}_{x}\left(\operatorname{exh}_{1}[A x \vee B x]\right)\right)= \\
& \operatorname{EVERY}_{x}(A x \vee B x) \wedge \neg\left(\operatorname{EVERY}_{x}(A x \wedge \neg B x)\right) \wedge\left(\operatorname{EVERY}_{x}(A x \wedge \neg B x)\right)= \\
& \operatorname{EVERY}_{x}(A x \vee B x) \wedge \operatorname{SOME}_{x}(A x) \wedge \operatorname{SOME}_{x}(B x) \\
\text { (62) } & \operatorname{Alt}_{1}=\{A x \vee B x, A x, B x\} \\
\text { (63) } & \operatorname{Alt}_{2}=\left\{\begin{array}{l}
\operatorname{EVERY}_{x}(\operatorname{exh}[A x \vee B x])=\operatorname{EVERY}_{x}(A x \vee B x) \\
\operatorname{EVERY}_{x}(\operatorname{exh}(A x))=\operatorname{EVERY}_{x}(A x \wedge \neg B x) \\
\operatorname{EVERY}_{x}(\operatorname{exh}(B x))=\operatorname{EVERY}_{x}(B x \wedge \neg A x)
\end{array}\right\}
\end{array}
$$

In sum, we have shown that all plausible parses based on non-replacement alternatives either lead to the distributive or the conjunctive inference or no inference at all. We next consider possible parses when the conjunctive alternative is assumed to be available. The outcome is analogous: the resulting parses are associated with either the distributive inference or one of the two inferences in (49) and (50), neither of which, as we have previously mentioned, can account for our results.

\section{D.2 Parses based on availability of all alternatives}

We describe the parses below and how they relate to our results.

- With only one exh at the global level, we once again derive the distributive inferences. The negation of the conjunctive alternative, corresponding to (49) above, is already entailed by the negation of the other two alternatives. The derivation is therefore the same as above.

$$
\begin{aligned}
& \operatorname{exh}\left(\operatorname{EVERY}_{x}(A x \vee B x)\right)= \\
& \operatorname{EVERY}_{x}(A x \vee B x) \wedge \neg \operatorname{EVERY}_{x}(A x) \wedge \neg \operatorname{EVERY}_{x}(B x)= \\
& \operatorname{EVERY}_{x}(A x \vee B x) \wedge \operatorname{some}_{x}(A x) \wedge \operatorname{SOME}_{x}(B x) \\
& \text { Alt }=\left\{\begin{array}{l}
\operatorname{EVERY}_{x}(A x \vee B x) \\
\operatorname{EVERY}_{x}(A x) \\
\operatorname{EVERY}_{x}(B x) \\
\operatorname{EVERY}_{x}(A x \wedge B x)
\end{array}\right\}
\end{aligned}
$$

- Having two global exh's leads again to the distributive inferences. It is straightforward to show that the second exh does not add anything, regardless of the presence of the conjunctive alternative. We therefore do not repeat the derivation here. 
- Having one local exh leads to the inference in (50), given that the conjunctive alternative is locally excludable.

(66) $\operatorname{EVERY}_{x}[\operatorname{exh}[A x \vee B x]]=\operatorname{EVERY}_{x}[(A x \vee B x) \wedge \neg(A x \wedge B x)]$

(67) Alt $=\{(A x \vee B x), A x, B x,(A x \wedge B x)\}$

- Having two local exh's results in the same inference in (50), as the second exh does not add anything. This is because the conjunctive alternative makes it so that the alternatives corresponding to each disjunct are nonexcludable in the second round of exhaustification.

(68) $\operatorname{EVERY}_{x}\left[\operatorname{exh}_{2}\left[\operatorname{exh}_{1}[A x \vee B x]\right]\right]=$ $\operatorname{EVERY}_{x}[(A x \vee B x) \wedge \neg(A x \wedge B x)]$

(69) $\mathrm{Alt}_{1}=\{(A x \vee B x), A x, B x,(A x \wedge B x)\}$

(70) $\mathrm{Alt}_{2}=\{((A x \vee B x) \wedge \neg(A x \wedge B x)), A x \wedge \neg B x, B x \wedge \neg A x,(A x \wedge B x)\}$

- Having one local exh and one global exh also leads to the same inference. This is again because this inference blocks the negation of the alternatives corresponding to the disjuncts in the second round of exhaustification (see also Crnič et al. 2015 for discussion).

(71) $\operatorname{exh}_{2}\left(\operatorname{EVERY}_{x}\left(\operatorname{exh}_{1}[A x \vee B x]\right)\right)=$ EVERY $_{x}[(A x \vee B x) \wedge \neg(A x \wedge B x)]$

$$
\begin{aligned}
& \operatorname{Alt}_{1}=\{A x \vee B x, A x, B x, A x \wedge B x\} \\
& \operatorname{Alt}_{2}=\left\{\begin{array}{l}
\operatorname{EVERY}_{x}(\operatorname{exh}[A x \vee B x])=\operatorname{EVERY}_{x}[(A x \vee B x) \wedge \neg(A x \wedge B x)] \\
\operatorname{EVERY}_{x}(\operatorname{exh}(A x))=\operatorname{EVERY}_{x}(A x \wedge \neg B x) \\
\operatorname{EVERY}_{x}\left(\operatorname{exh}(B x)=\operatorname{EVERY}_{x}(B x \wedge \neg A x)\right. \\
\operatorname{EVERY}_{x}(\operatorname{exh}(A x \wedge B x))=\operatorname{EVERY}_{x}(A x \wedge B x)
\end{array}\right\}
\end{aligned}
$$

In sum, whether or not the conjunctive alternative is assumed to be available, no plausible inferences other than the distributive and conjunctive inferences can explain the observed pattern of results.

\section{Appendix E: Children's justifications for no-responses}

In this Appendix, we report the justifications that children gave for providing no-responses, for both the DISTRIBUTIVE and NON-DISTRIBUTIVE conditions.

\begin{tabular}{l|l|l} 
ID & Condition & Justification \\
\hline Child1 & NON-DISTRIBUTIVE & $\begin{array}{l}\text { Everyone opened a big can. Her said everyone } \\
\text { opened a big one and a small one. }\end{array}$ \\
\hline Child1 & NON-DISTRIBUTIVE & Everyone drinked a big juice. \\
\hline
\end{tabular}




\begin{tabular}{|c|c|c|}
\hline Child1 & NON-DISTRIBUTIVE & Everyone ate a big cookie only. \\
\hline Child1 & NON-DISTRIBUTIVE & Everyone did a big dish, not a small dish too. \\
\hline Child1 & DISTRIBUTIVE & $\begin{array}{l}\text { One got a little butterfly, and two more have } \\
\text { the big butterflies. }\end{array}$ \\
\hline Child1 & DISTRIBUTIVE & Two have big teas, and one got a little tea. \\
\hline Child1 & DISTRIBUTIVE & $\begin{array}{l}\text { Two girls have a big pizza, and one got a little } \\
\text { pizza. }\end{array}$ \\
\hline Child1 & DISTRIBUTIVE & One ride a little car, and two ride the big cars. \\
\hline Child2 & NON-DISTRIBUTIVE & They opened a big can. \\
\hline Child2 & NON-DISTRIBUTIVE & All of the mouse drank a \\
\hline Child2 & NON-DISTRIBUTIVE & They ate a big cookie. \\
\hline Child2 & NON-DISTRIBUTIVE & They washed a big dish. \\
\hline Child3 & NON-DISTRIBUTIVE & All of the mice drank big juices. \\
\hline Child3 & NON-DISTRIBUTIVE & All of the cows just washed a big dish. \\
\hline Child3 & DISTRIBUTIVE & $\begin{array}{l}\text { Two caught big butterflies and one caught a lit- } \\
\text { tle butterfly. }\end{array}$ \\
\hline Child3 & DISTRIBUTIVE & Two bought big teas and one bought a little tea. \\
\hline Child3 & DISTRIBUTIVE & Two ate big ones and one ate little one. \\
\hline Child3 & DISTRIBUTIVE & Two drove big cars and one drove a little car. \\
\hline Child4 & NON-DISTRIBUTIVE & They got a big can. \\
\hline Child4 & NON-DISTRIBUTIVE & They got a big juice. \\
\hline Child4 & NON-DISTRIBUTIVE & They got a big cookie. \\
\hline Child4 & NON-DISTRIBUTIVE & They did a big dish. \\
\hline Child5 & NON-DISTRIBUTIVE & They all opened a big can. \\
\hline Child5 & NON-DISTRIBUTIVE & They only ate a big cookie. \\
\hline Child5 & NON-DISTRIBUTIVE & Every cow didn't do little dish. \\
\hline Child6 & NON-DISTRIBUTIVE & Every cat opened a big one. \\
\hline Child6 & NON-DISTRIBUTIVE & Everyone drank a big one. \\
\hline Child 6 & NON-DISTRIBUTIVE & Everyone ate a big one. \\
\hline Child6 & NON-DISTRIBUTIVE & They washed big dish. \\
\hline Child6 & DISTRIBUTIVE & One buyed a small one. \\
\hline Child6 & DISTRIBUTIVE & One drank a small tea. Two drank big tea. \\
\hline Child6 & DISTRIBUTIVE & One girl ate a small one. \\
\hline Child6 & DISTRIBUTIVE & One drived a yellow, small car. \\
\hline Child7 & NON-DISTRIBUTIVE & They only did the big dishes. \\
\hline Child 8 & DISTRIBUTIVE & Only one got a small butterfly. \\
\hline Child8 & DISTRIBUTIVE & Only one got a small tea. \\
\hline Child8 & DISTRIBUTIVE & Only one of them ate a small pizza. \\
\hline Child8 & DISTRIBUTIVE & Only one drove a small car. \\
\hline Child9 & DISTRIBUTIVE & $\begin{array}{l}\text { One caught a small butterfly. Two caught a big } \\
\text { butterfly. }\end{array}$ \\
\hline Child9 & DISTRIBUTIVE & One drank a small tea. Two drank a big tea. \\
\hline Child9 & DISTRIBUTIVE & One ate small, two big pizza. \\
\hline
\end{tabular}




\begin{tabular}{l|l|l}
\hline Child9 & DISTRIBUTIVE & Two drove a big car and one drove a small car. \\
\hline Child10 & NON-DISTRIBUTIVE & The cats all got one big can. \\
\hline Child10 & NON-DISTRIBUTIVE & $\begin{array}{l}\text { All of the mice drinked orange juice, lemon juice, } \\
\text { and apple juice. }\end{array}$ \\
\hline Child10 & NON-DISTRIBUTIVE & All the fairies have a big cookie. \\
\hline Child10 & NON-DISTRIBUTIVE & All the cows have washed one big dish. \\
\hline Child11 & NON-DISTRIBUTIVE & They only opened a big can. \\
\hline Child11 & NON-DISTRIBUTIVE & They all drank a big juice. \\
\hline Child11 & NON-DISTRIBUTIVE & The ate all a big cookie. \\
\hline Child11 & NON-DISTRIBUTIVE & They all did wash a big dish. \\
\hline Child12 & NON-DISTRIBUTIVE & Every cat opened a big can. \\
\hline Child12 & NON-DISTRIBUTIVE & Every mice have a big juice. \\
\hline Child12 & NON-DISTRIBUTIVE & All the fairies ate a big cookie. \\
\hline Child13 & NON-DISTRIBUTIVE & Every cow washed a big dish. \\
\hline Child13 & NON-DISTRIBUTIVE & All of them opened the big ones. \\
\hline Child13 & NON-DISTRIBUTIVE & All of them had the big one. \\
\hline Child13 & NON-DISTRIBUTIVE & All wanted the big ones. \\
\hline Child13 & DISTRIBUTIVE & $\begin{array}{l}\text { Because the first one said the big ones and the } \\
\text { other one said the little one. }\end{array}$ \\
\hline Child13 & DISTRIBUTIVE & $\begin{array}{l}\text { Because the first one said the little one, and the } \\
\text { others said the big ones. }\end{array}$ \\
\hline Child13 & DISTRIBUTIVE & $\begin{array}{l}\text { Because the two girls said the big pizza and one } \\
\text { said the small one. }\end{array}$ \\
\hline Child13 & DISTRIBUTIVE & $\begin{array}{l}\text { The first one said I want to drive a big car, and } \\
\text { the other one said big car, and the other one } \\
\text { said small car. }\end{array}$ \\
\hline Child14 & NON-DISTRIBUTIVE & They drank big ones. \\
\hline Child15 & NON-DISTRIBUTIVE & Because all the cats opened a big can. \\
\hline Child15 & NON-DISTRIBUTIVE & Because all the mouse drank a big juice. \\
\hline Child15 & DON-DISTRIBUTIVE & $\begin{array}{l}\text { Because all the fairies ate a big one. } \\
\text { All of the cows washed a big dish. }\end{array}$ \\
\hline & $\begin{array}{l}\text { Because two dogs drove a big car and one dog } \\
\text { drove a little car. }\end{array}$ \\
\hline
\end{tabular}




\section{References}

Alonso-Ovalle, L. (2005). Distributing the disjuncts over the modal space. In PROCEEDINGS-NELS, volume 35, page 75 .

An, S. (2015). The acquisition of constraints in child mandarin. PhD thesis, Macquarie University.

Barker, C. (2010). Free choice permission as resource-sensitive reasoning. Semantics and Pragmatics, 3:10-1.

Barner, D. and Bachrach, A. (2010). Inference and exact numerical representation in early language development. Cognitive psychology, 60(1):40-62.

Barner, D., Brooks, N., and Bale, A. (2011). Accessing the unsaid: The role of scalar alternatives in children's pragmatic inference. Cognition, 118(1):84-93.

Barr, D. J. (2013). Random effects structure for testing interactions in linear mixed-effects models. Frontiers in psychology, 4.

Bill, C., Romoli, J., Schwarz, F., and Crain, S. (2016). Scalar implicatures versus presuppositions: The view from acquisition. Topoi, 35(1):57-71.

Boster, C. and Crain, S. (1993). On children's understanding of every and or. In Proceedings of early cognition and transition to language.

Bowler, M. (2014). Conjunction and disjunction in a language without'and'. In Semantics and Linguistic Theory, volume 24, pages 137-155.

Braine, M. D. and Rumain, B. (1981). Development of comprehension of or: Evidence for a sequence of competencies. Journal of experimental child psychology, 31(1):46-70.

Breheny, R., Klinedinst, N., Romoli, J., and Sudo, Y. (2016). The symmetry problem: current theories and prospects. under review, available at https://www.ucl.ac.uk/ ucjtudo/pdf/alternatives.pdf.

Chemla, E. (2009). Similarity: Towards a unified account of scalar implicatures, free choice permission and presupposition projection. Under revision for Semantics and Pragmatics.

Chemla, E. and Bott, L. (2014). Processing inferences at the semantics/pragmatics frontier: disjunctions and free choice. Cognition, 130(3):380396.

Chierchia, G. (2006). Broaden your views: Implicatures of domain widening and the "logicality" of language. Linguistic inquiry, 37(4):535-590.

Chierchia, G. (2013). Logic in grammar: Polarity, free choice, and intervention. OUP Oxford. 
Chierchia, G., Crain, S., Guasti, M. T., Gualmini, A., and Meroni, L. (2001). The acquisition of disjunction: Evidence for a grammatical view of scalar implicatures. In Proceedings of the 25th Boston University conference on language development, pages 157-168. Cascadilla Press Somerville, MA.

Chierchia, G., Guasti, M. T., Gualmini, A., Meroni, L., Crain, S., and Foppolo, F. (2004). Semantic and pragmatic competence in childrens and adults comprehension of or. In Experimental pragmatics, pages 283-300. Springer.

Crain, S. and Thornton, R. (1998). Investigations in universal grammar. Cambridge, MA: MIT Press.

Crnič, L., Chemla, E., and Fox, D. (2015). Scalar implicatures of embedded disjunction. Natural Language Semantics, 23(4):271-305.

Davidson, K. (2013). And'or 'or': General use coordination in asl. Semantics Es Pragmatics, 6(4):1-44.

Foppolo, F., Guasti, M. T., and Chierchia, G. (2012). Scalar implicatures in child language: Give children a chance. Language learning and development, 8(4):365-394.

Fox, D. (2007). Free choice and the theory of scalar implicatures. In Presupposition and implicature in compositional semantics, pages 71-120. Springer.

Fox, D. and Katzir, R. (2011). On the characterization of alternatives. Natural Language Semantics, 19(1):87-107.

Gamut (1991). Logic, Language and Meaning. University of Chicago Press.

Gazdar, G. (1979). Pragmatics: Presupposition, Implicature, and Logical Form. New York: Academic Press.

Geurts, B. (2005). Entertaining alternatives: Disjunctions as modals. Natural language semantics, 13(4):383-410.

Grice, H. P. (1975). Logic and conversation. 1975, pages 41-58.

Gualmini, A., Crain, S., Meroni, L., Chierchia, G., and Guasti, M. T. (2001). At the semantics/pragmatics interface in child language. In Proceedings of SALT XI, pages 231-247. Citeseer.

Guasti, M. T., Chierchia, G., Crain, S., Foppolo, F., Gualmini, A., and Meroni, L. (2005). Why children and adults sometimes (but not always) compute implicatures. Language and cognitive processes, 20(5):667-696.

Hochstein, L., Bale, A., Fox, D., and Barner, D. (2016). Ignorance and inference: do problems with gricean epistemic reasoning explain children's difficulty with scalar implicature? Journal of Semantics, 33(1):107-135. 
Horn, L. R. (1972). On the semantic properties of logical operators in English. $\mathrm{PhD}$ thesis, University of California, Los Angeles.

Huang, A. and Crain, S. (2014). Acquisition of the polarity sensitive item renhe 'any'in mandarin chinese. Journal of child language, 41(4):861-889.

Huang, Y. T., Spelke, E., and Snedeker, J. (2013). What exactly do numbers mean? Language Learning and Development, 9(2):105-129.

Jaeger, T. F. (2008). Categorical data analysis: Away from anovas (transformation or not) and towards logit mixed models. Journal of memory and language, 59(4):434-446.

Katsos, N. and Bishop, D. V. (2011). Pragmatic tolerance: Implications for the acquisition of informativeness and implicature. Cognition, 120(1):67-81.

Katzir, R. (2007). Structurally-defined alternatives. Linguistics and Philosophy, 30(6):669-690.

Kiguchi, H. and Thornton, R. (2016). Connectivity effects in pseudoclefts in child language. Studia linguistica, 170(1):34-65.

Klinedinst, N. W. (2007). Plurality and possibility. PhD thesis, Citeseer.

Kratzer, A. and Shimoyama, J. (2002). Indeterminate pronouns: The view from japanese. In Proceedings of the Third Tokyo Conference on Psycholinguistics.

Levinson, S. C. (2000). Presumptive meanings: The theory of generalized conversational implicature. MIT Press.

Meyer, M.-C. (2013). Ignorance and grammar. PhD thesis, Massachusetts Institute of Technology.

Noveck, I. A. (2001). When children are more logical than adults: Experimental investigations of scalar implicature. Cognition, 78(2):165-188.

Papafragou, A. and Musolino, J. (2003). Scalar implicatures: experiments at the semantics-pragmatics interface. Cognition, 86(3):253-282.

Paris, S. G. (1973). Comprehension of language connectives and propositional logical relationships. Journal of experimental child psychology, 16(2):278-291.

Philip, W. (1991). Quantification over events in early universal quantification. In 16th annual Boston University Conference on Language Development, Boston, MA.

Philip, W. (1995). Event quantification in the acquisition of universal quantification. PhD thesis, University of Massachusetts.

Rakhlin, N. (2007). A new pragmatic account of quantifier-spreading. Nanzan Linguistics, 3:239-282. 
Reinhart, T. (2006). Interface Strategies. Optimal and Costly Derivations. Cambridge, Mass.: MIT Press.

Romoli, J. (2013). A scalar implicature-based approach to neg-raising. Linguistics and philosophy, 36(4):291-353.

Sauerland, U. (2004). Scalar implicatures in complex sentences. Linguistics and philosophy, 27(3):367-391.

Singh, R., Wexler, K., Astle-Rahim, A., Kamawar, D., and Fox, D. (2016). Children interpret disjunction as conjunction: Consequences for theories of implicature and child development. Natural Language Semantics, 24(4):305352 .

Skordos, D. and Papafragou, A. (2016). Children's derivation of scalar implicatures: Alternatives and relevance. Cognition, 153:6-18.

Spector, B. (2007). Aspects of the pragmatics of plural morphology: On higherorder implicatures. In Presupposition and implicature in compositional semantics, pages 243-281. Springer.

Stiller, A. J., Goodman, N. D., and Frank, M. C. (2015). Ad-hoc implicature in preschool children. Language Learning and Development, 11(2):176-190.

Sugisaki, K. and Isobe, M. (2001). Quantification without qualification without plausible dissent. University of Massachusetts Occasional Papers in Linguistics, 25:97-100.

Thomas, G. G. P. Y. (2012). Temporal implicatures. PhD thesis, Massachusetts Institute of Technology.

Tieu, L., Bill, C., Romoli, J., and Crain, S. (2014). Plurality inferences are scalar implicatures: Evidence from acquisition. In Semantics and Linguistic Theory, volume 24, pages 122-136.

Tieu, L., Romoli, J., Zhou, P., and Crain, S. (2016). Children's knowledge of free choice inferences and scalar implicatures. Journal of Semantics, 33(2):269298.

Tieu, L., Yatsushiro, K., Cremers, A., Romoli, J., Sauerland, U., and Chemla, E. (2017). On the role of alternatives in the acquisition of simple and complex disjunctions in french and japanese. Journal of Semantics, 34(1):127-152.

Trinh, T. and Haida, A. (2015). Constraining the derivation of alternatives. Natural Language Semantics, 23(4):249-270.

Zhou, P., Romoli, J., and Crain, S. (2013). Children's knowledge of free choice inferences. In Semantics and Linguistic Theory, volume 23, pages 632-651.

Zimmermann, T. E. (2000). Free choice disjunction and epistemic possibility. Natural language semantics, 8(4):255-290. 